\title{
A needs analysis for communication by pharmacists in a multilingual setting: First steps towards syllabus and materials design
}

Kris van de Poel

University of Antwerp, Belgium | School of Languages, Potchefstroom Campus, North-West University, South Africa

E-mail: kris.vdpoel@telenet.be

Tobie van Dyk

Centre for Academic and Professional Language Practice, School of Languages, Potchefstroom Campus, NorthWest University, South Africa

E-mail: tobie.vandyk@nwu.ac.za

Jessica Gasiorek

University of Hawai'i at Mānoa, USA | School of Languages, Potchefstroom Campus, North-West University, South Africa

E-mail: gasiorek@hawaii.edu

Inge GE Blockmans

PhD Fellowship of the Research Foundation - Flanders, Ghent University and KU Leuven, Belgium

E-mail: ingeblockmans@hotmail.com

\begin{abstract}
Despite the efforts to manage South Africa's language and culture diversity, in practice, the linguistic landscape has become increasingly English. This is problematic in contexts such as rural areas where people are not able to communicate in English, and communication on even a fairly proficient level is not a given. Medical contexts, in particular, may create critical situations of practical (life or death) importance, as healthcare professionals are not necessarily trained or equipped to communicate in an efficient and culturally sensitive manner. Moreover, healthcare communication challenges are manifold because of the diverse views on the nature of a medical encounter. This article reports on the first empirical steps taken to bridge the communication gap between patient and medical practitioner (specifically pharmacists). These steps include identifying an appropriate theoretical framework, developing and conducting a needs analysis (among 255 pharmacists who are experts-by-experience), and designing a syllabus and course materials. The findings show that most respondents experience communication in a foreign language to be significantly more problematic than communication in general. Additionally, the qualitative data suggest that support is needed especially for African languages. Language learning materials should cover a wide range of professional topics supplemented with a communication module focusing primarily on history taking and
\end{abstract}


ensuring the correct use of medication. Most importantly, learning materials should be to-thepoint and easy to apply, as most practitioners have limited time. These findings might, in turn, inform policy issues regarding responsible and efficient functioning in a multilingual professional environment, where clear and transparent communication can be a matter of life or death.

Keywords: needs analysis, healthcare, professional communication, communication training

\section{Introduction: Context and problem}

South Africa is a multilingual country with 11 official languages, all of which have the same status, rights and privileges as stipulated in the Constitution of the Republic of South Africa (South African Government 1997). Since 1994, the South African Government has made progress in providing a framework and establishing bodies to manage language diversity in the country. However, the South African linguistic landscape has become increasingly English over the last 20 years. Whereas English is often considered to be a lingua franca, it is only the fourth-largest language in South Africa and is spoken by less than $50 \%$ of the population, with isiZulu being the largest, followed by isiXhosa and Afrikaans as the second and third most popular languages, respectively, in South African households (South African Government 2013).

Communication at a proficient level is thus not necessarily a given, and service encounters may be highly challenging in, for instance, rural areas and/or healthcare settings where clients/patients are unable to communicate in English. When considering the service providers' angle, where most professionals are proficient in Afrikaans and/or English, they are not necessarily trained or equipped to communicate in an efficient and culturally sensitive manner, nor are they proficient in one or more of the African languages (cf. the recent introduction of language learning in the healthcare curriculum). Moreover, in a healthcare setting, challenges include not only the number of languages and cultures involved, but also "the diverse views on the nature of a medical encounter" (Van de Poel and Fourie 2013: 333), coming to the fore in, for example, the relationship between the healthcare professional and the patient, the interpretation of the interactants' status and power, and the interpretation of the sources and nature of illness.

This complex nature of healthcare communication was recognised by the Faculty of Health Sciences and the Centre for Academic and Professional Language Practice of North-West University's (NWU) Potchefstroom Campus. Consequently, with collaboration and support from the University of Antwerp and the Communication for Professionals network (cf. Communication for Professionals 2014), it was decided to address this matter by developing multilingual ${ }^{1}$ blended vocational language and communication training materials for students registered for the BPharm (Pharmacy) qualification. The ultimate goal was to develop course materials that will enhance efficient functioning in a multilingual work environment where adequate, accurate and appropriate communication is of critical importance. Hence, the guiding question for this study was: How can programme and materials design be informed by theory and by drawing on pharmacists as expertsby-experience in intercultural professional communication?

\footnotetext{
${ }^{1}$ A template for materials development was developed in English and was used as a basis for materials in other languages (Afrikaans and Setswana as the other official languages of NWU, as well as isiZulu and isiXhosa).
} 
The purpose of this article is to report on the initial steps taken in the course design and materials development process which comprised the identification of an appropriate theoretical framework as well as an in-depth needs analysis. The report will also signify how the course design was informed by theory and needs.

\section{Theoretical framework}

The domain of English for Specific Purposes (ESP) was deemed to be an appropriate conceptual point of departure. The reason for this is that ESP, by definition, typically attempts to meet the identifiable needs of learners, makes use of the underlying practices of the disciplines it serves, and concentrates on language suitable to the specific environment in terms of lexis, grammar, style, genre and discourse (Dudley-Evans 2001). In what is to follow, a more systematic and detailed explication of ESP will be presented.

\subsection{English language learning and training for specific purposes}

ESP is divided into two major subdomains (Carkin 2005). First, English for Academic Purposes (EAP) concerns itself with English used in academic contexts and more specifically with the genres involved. It focuses on "learning academic language through academic tasks, texts, and content" and it encompasses "needs analyses, evaluation, academic skills, disciplinary content, and tasks in support of student learning in tertiary educational contexts" (Carkin 2005: 85). English for Occupational Purposes (EOP) is the second subdomain of ESP and includes "English language used by both professionals (for instance, in medicine, business, law) and by non-professional workers (in vocational ${ }^{2}$ contexts)" (Carkin 2005: 85).

Another important notion closely associated with ESP is 'intercultural communication'. ESP teachers and learners are not only involved with teaching or learning language, but also with the development of intercultural competence and acculturation. The success of an ESP course does not only depend on the learner's proficiency in the target language, but also on their ability to integrate successfully in a new work environment or study culture (see Fatihi 2003, Warrington 2005). Thus, the ESP-learner may benefit substantially from an emphasis on intercultural knowledge and skills (Berbyuk-Lindström 2008). However, as acculturation is a highly sensitive and individual process, incorporating intercultural content which will serve all learners in one class complicates the design of an ESP course.

\subsection{Language for specific purposes}

Since many professionals nowadays move between cultures, these professionals often need more than English as a lingua franca or as a language for wider communication. In other words, they need to acquire (some of) the specific language of the community in which they are working. The language to be learned, therefore, is not only a language for specific purposes; it is also a language for intercultural communication. In these instances, the term "ESP" is broadened to Language for Specific Purposes (LSP; Gatehouse 2001) which may take the form of an additional language and remain restricted to particular domains or functions.

\footnotetext{
${ }^{2}$ We use the term "vocational" in our article to refer to professional communication in general.
} 
LSP teaching (being contact teaching, autonomous learning or blended learning) aims to fulfil the communicative needs of speakers when facing a particular academic or professional context. The courses aim "to help the learners become better equipped linguistically to cope with the communicative demands they face in their work or study situation" (Basturkmen and Elder 2006: 672). The importance of the learners' needs is one of the defining characteristics of LSP, as materials and courses are based on the results of a needs analysis (Brown 1995, Dudley-Evans 2001). Moreover, as LSP teaching and syllabus design often arise from pressing situations, they are "driven largely by practical rather than theoretical concerns" (Basturkmen and Elder 2006: 673).

There are a number of key issues in the development of an LSP course (Gatehouse 2001). Several abilities are required for successful communication in occupational settings. As a result, LSP programmes usually outline the needs of learners so as to provide them with the jargon of their context as well as to offer more general language content. However, the balance between general and specific proficiency has to be determined. It should be noted that there can be overlap in how these two considerations are addressed; also, the needs of individual language learners can differ. In addition to language or jargon use specific to their occupation, LSP-learners often require a set of more general skills which feed communicative competence, such as how to write a report or how to chair a meeting. These skills often help or allow language-discordant professionals - often people who lack proficiency in the language(s) of their professional environment - to integrate into the workforce, to establish social relationships, and to be more open to lifelong learning. Material developers need to incorporate these features in accordance with the ultimate goal of designing a language and communication course that will contribute to efficiency in a multilingual work environment. Thus, a needs analysis and careful examination of the occupational context needs to precede materials design.

\subsubsection{Towards designing a syllabus for specific needs}

Professional learners are often identified as a heterogeneous group. Being who they are, they will have to be presented with diversified, broad, easily accessible, adequate and appropriate content which reflects their professional context as closely as possible (Ali and Salih 2013). This brings about a number of challenges which have to be taken into account when designing a LSP programme, namely the inclusion of low-level proficiency learners, the integration of several skills in the course materials, the creation of a platform where a balance between content knowledge and general language knowledge is achieved, as well as assurance that the relevant voices are heard and their needs addressed (Basturkmen and Elder 2006: 677).

\subsubsection{Towards materials development for healthcare professionals}

Since LSP learning often takes place when the learner is already engaged in the professional context, the learner may not optimally benefit from conventional contact teaching. Professionals in mobile contexts - i.e. professionals who are not bound to one working environment or region - have relatively little time for contact teaching and, as a result, materials are often made available for autonomous learning. However, irrespective of whether the materials are printed or available electronically (i.e. accessible online or via mobile phone), learners often do not know how to deal with these materials independently and thus require support (Van de Poel and Fourie 2013). To this end, blended learning (Bonk and Graham 2006) 
can be a solution, where online autonomous learning can be supported by contact sessions with a teacher, expert, monitor, tutor or peer.

\section{Research design}

As indicated earlier, the present study was carried out as a first step to inform the language and communication materials design process for pharmacists in South Africa. Thus, within the theoretical framework of the study, the actual needs of practicing pharmacists had to be identified. In the empirical part of the study, a needs analysis investigated the different roles pharmacists have in their professional contexts, the communicative challenges they face, and their perceived language-learning needs. These data were then mapped onto the curricular needs formulated by the Faculty of Health Sciences at NWU, and transformed into a syllabus as a foundation for online/mobile materials development. The next section will briefly introduce the concept of 'needs analysis'. The following section will then present and discuss the empirical investigation.

\subsection{Needs analysis}

Berwick (1989: 56) argues that a need refers to a gap between what is and what should/could be. In order to overcome this gap in syllabus and course design, one should be well informed about the true needs, challenges and interests of students/learners. Data to this end are typically gathered by means of a needs analysis. Brown (1995: 35) refers to a needs analysis as "the activities involved in [systematically] gathering [both subjective and objective] information that will serve as the basis for [defining, developing and validating] a [defensible syllabus] that will meet the learning needs of a particular group of students". This is confirmed by Richards (2001). A needs analysis is a powerful tool that clarifies, verifies and validates the true needs of students/learners and not only the perceived needs as determined by, e.g. language practitioners. Indeed, it has the power to shape syllabi and learning materials (course design), and it warrants the needs, gaps and wants or interests of students/learners (Lepetit and Cichocki 2002; Long 2005).

According to Hutchinson and Waters (1987: 55), needs can be divided into target needs and learning needs. Target needs are what a person should be able to do in the target language use situation: (i) what the student/learner needs to know to function effectively in a particular context; (ii) what hindrances the student/learner will face in this particular context; and (iii) what the student/learner sees as important or interesting and wants to learn. Learning needs are what the student/learner needs to do in order to learn. In keeping with Hutchinson and Waters (1987), the learning needs should also be reflected by the learning route (materials), and not only the target needs as is often the case. For the purposes of this study, both the target and learning needs inventories were informed by Gasiorek and Van de Poel (2012) and Van de Poel, Vanagt, Schrimpf and Gasiorek (2013), relying on Basturkmen and Elder (2006), DudleyEvans (2001), Flowerdew (2013) and Gatehouse (2001), among others, and taking into account the insights presented in the work by Beardsley, Kimberlin and Tindall (2012) and McDonough and Bennett (2006). In what follows, we will describe the in-depth needs analysis conducted for this study. In the final section, the course design, informed by theory and needs, will be outlined. 


\subsection{Empirical investigation}

\subsubsection{Instruments and procedure}

As indicated above, the original Medics on the Move needs analysis instrument (reported in Gasiorek and Van de Poel 2012; Van de Poel 2009 and 2011; Van de Poel and Brunfaut 2010; Van de Poel and Gasiorek 2012a and 2012b; Van de Poel, Vanagt, Schrimpf and Gasiorek 2013) was adapted and extended to target pharmacist-specific topics as well as the South African context. The changes made for this new context were based on intercultural communication research and the expertise of qualified South African pharmacists.

The questionnaire used to gather information included a needs inventory with two components containing both closed and open-ended questions. ${ }^{3}$ The first component (the communication component) asked participants about their professional communication experiences, expectations and needs. The second or pedagogical component consisted of three questions which explored the participants' needs and wishes with regard to communication training and learning. These questions occasionally overlapped with those in the communication component, allowing for verification of responses.

The questionnaire was piloted for validation purposes among staff at the School of Pharmacy at NWU in September 2013. In October 2013, an online invitation in five languages (English, Afrikaans, Setswana, isiXhosa, Sesotho) and a link to the (English) survey was sent to 10,602 email accounts kept at the official NWU registry as well as 40 NWU staff members at the School of Pharmacy, and 64 Master's and $\mathrm{PhD}$ students enrolled in this particular school. After two months, the total number of complete responses (i.e. responses to all questions) was 255 , representing a response rate of only $2 \%{ }^{4}$. The low response rate may be due to the extent of applicability of the survey to the addressees' working environments, lack of interest, lack of time, passive e-mail accounts, the rather academic tone of the invitation and the questionnaire, the fact that the survey was only available in English, amongst other things. Despite the length of the survey, the respondents appeared to have given detailed responses and even sent the researchers grateful, positive feedback upon completion of the survey. Although the response rate shows a low percentage, it is nevertheless a fair-sized sample. All respondents gave informed consent for the data to be used.

\subsubsection{Participants}

As noted above, 255 pharmacists voluntarily completed the online questionnaire as far as it was applicable to their professional situation. The gender distribution (69.6\% females, $30.4 \%$ males) is generally representative of the pharmaceutical professional population in South Africa (South African Government 2013). Of the respondents, $40.8 \%$ were over 50 years old, $26.6 \%$ were between 30 and 40 years of age, and roughly $20 \%$ were between 40 and 50 years of age. The remainder of the participants were younger than 30 (25-30: 9.2\%; 20-25: 1.0\%).

In terms of professional background, the participants had on average 20.29 years of experience in pharmacy $(\mathrm{SD}=12.23)$. Most respondents were practitioners and thus worked in a pharmacy

\footnotetext{
${ }^{3}$ Additional information was also gathered with this questionnaire, but it is outside the scope of this paper, and therefore will not be reported or discussed here.

${ }^{4}$ Note that approximately 1,600 emails were returned as undeliverable and calculations were adapted accordingly.
} 
(76.5\%), followed by $15.8 \%$ who selected "other" (e.g. active in research, the pharmaceutical industry, and/or in pharmaceutical consultancy). Finally, some academic staff (6.6\%) and two students $(1 \%)$ also participated. Approximately one third $(30.6 \%)$ of participants reported that they were currently employed in retail pharmacies. The remaining participants reported being employed in "other" situations (e.g. regulation, administration, consultancy (21.9\%); industrial pharmacy $(11.7 \%)$; private hospital $(11.7 \%)$; government sector $(11.2 \%)$; research and education (6.1\%); and education and sales $(0.5 \%)$ ).

With regard to language use ${ }^{5}$, almost half of the participants use Afrikaans as their first language (46.43\%), while English is the first language of 39.8\% of the participants. The other languages are distributed in the following way: Sesotho $(2.55 \%)$, isiXhosa $(1.02 \%)$ or isiZulu, Shona, Tshivenda, Xitsonga, Dutch, or German (all 0.51\%). Most respondents (89.8\%) claimed to speak as a second language: English (58.52\%), Afrikaans (36.93\%), isiXhosa $(1.70 \%)$, or Gujarati, Italian, Sesotho, isiZulu (all 0.57\%). A considerable number (26.02\%) speak a third language, and some a fourth language $(12 \%)$.

Two-thirds of the respondents $(67.86 \%)$ use English as their primary professional language, followed by Afrikaans (21.94\%) or both (0.51\%). For four respondents $(2.04 \%)$, another language was dominant in professional contexts (Sesotho sa Leboa, Xitsonga, isiZulu). Participants reported speaking these dominant languages for 38 hours per week on average. Just under three-quarters (72.45\%) reported using a second professional language: Afrikaans (53.52\% of 142 respondents, same sample for the percentages to follow here), English $(31.69 \%)$, Zulu (5.63\%), isiXhosa $(1.41 \%)$, or isiNdebele, Sesotho, Sesotho sa Leboa, Setswana, Dutch, Italian, Mandarin, Portuguese, or Russian (all 0.7\%). These second professional languages are spoken for 13 hours per week on average. A minority reported the use of a third professional language (16.8\%) and a fourth language (4.6\%), averaging 9 and 4 hours of use per week, respectively. Together, the respondents speak 10 of the 11 official languages: Afrikaans, English, isiZulu, isiXhosa, Setswana, Sesotho, Sesotho sa Leboa, Tshivenda, isiNdebele, and Xitsonga (thus all but siSwati). It should be noted, however, that most comments on these demographic questions indicated that knowledge of African languages is quite basic or non-existent: "ZULU IS VERY POOR", "I battle with Zulu [as second professional language]", "Swahili, just to help myself", "Wish I had learnt some Africann language", "Not very fluent in Zulu", "basic xhosa", "some knowledge of Xhosa, but very rusty", "Spoke Xhosa as a child, since lost it". 6

\section{Results and discussion}

\subsection{Communication}

The communication component of the needs inventory required respondents to indicate the following on a 5-point Likert scale (ranging from $1=$ "very problematic" to $5=$ "not problematic at all"):

\footnotetext{
5 The total sample size was used for calculating the percentages unless mentioned otherwise, even though 11 respondents did not answer the questions pertaining to language use.

${ }^{6}$ For greater legibility and focus on the ideas rather than the linguistic proficiency of the respondents, the responses are reproduced verbatim and have not been corrected.
} 
(i) To what extent they found "communication in general" problematic with patients/clients, colleagues, doctors and other professional contacts, and to specify or give an example (open-ended);

(ii) To what extent they found communication with the same people problematic "in a language other than [their] first language", to fill in which language they had in mind, and to give an "example of problematic communication in a language other than [their] first language";

(iii) To what extent they found several elements important whilst communicating with patients/clients (see Table 2 for a complete list);

(iv) To state whether they would consider the following as "barriers for conducting effective communication" in their profession: lack of interest, low priority, lack of skills, lack of knowledge, lack of resources, other (and if they selected the latter, to specify why).

\subsubsection{Communication in general is considered unproblematic}

In general, respondents ( $\mathrm{n}=154$ for these items) did not find communication in this context problematic ${ }^{7}$ with any group. As can be seen in Table 1, all means are well above the midpoint of the scale (where $1=$ "very problematic" and $5=$ "not problematic at all").

Table 1: Extent to which communication with different targets is considered problematic

\begin{tabular}{|c|c|c|c|c|c|c|}
\hline & \multicolumn{2}{|c|}{ General } & \multicolumn{2}{|c|}{$\begin{array}{l}\text { Second/Other } \\
\text { Language }\end{array}$} & \multirow[b]{2}{*}{$t(153)$} & \multirow[b]{2}{*}{$p$} \\
\hline & $M$ & $S D$ & $M$ & $S D$ & & \\
\hline Patients/clients & 3.84 & 1.07 & 3.31 & 1.24 & 6.85 & $<.001$ \\
\hline Colleagues & 4.38 & 0.79 & 3.74 & 1.16 & 7.21 & $<.001$ \\
\hline Doctors & 4.09 & 0.96 & 3.58 & 1.15 & 5.74 & $<.001$ \\
\hline Other professional contacts & 4.10 & 0.90 & 3.54 & 1.15 & 6.86 & $<.001$ \\
\hline
\end{tabular}

Open-ended responses indicated that problems arise from the lack of fluency in second and/or third languages ("especially with Xhosa-speaking patients", remarked one participant, although another indicated that there is "in general no problem if someone speaks Afrikaans or English"), scientific terminology, and different cultural and historical backgrounds (see Appendix $\mathrm{A}^{8} .1$ 3). Some respondents commented that when problems occur with patients, it is because: (i) "they [i.e. the patients] are usually uninformed or confused about conditions, medications, criteria for medication"; (ii) they do not speak/understand English or Afrikaans in rural areas; (iii) they attach different meanings to language possibly due to different health beliefs from their respective cultures (see Appendix A.4); or (iv) they are simply "difficult people" who do not "accept" the responses they get from the pharmacist (see Appendix A.5). Problems with doctors and specialists are ascribed to the limitation of their verbal skills to "areas within which they do not feel threatened" and hierarchy being a barrier to collaboration or the equal exchange of ideas: "communication with doctors is improving, but sometimes still hampered by personal pride vs. patient interest, e.g. confirming/questioning an antibiotic dose for a child and although

\footnotetext{
${ }^{7}$ Yet, as one respondent reported, "in [an] NGO setting [these] answers apply. In retail however different answers would apply."

${ }^{8}$ Appendix A contains the responses to open-ended questions and serves as qualitative data for the study to supplement the quantitative data reported in this section.
} 
having reason for concern, will be told to respect the doctor and not bother him/her with useless queries."

Notably, respondents also indicated that communication in a language different from their first language was not problematic. As with communication in general, means were all above the scale midpoint. However, four paired-samples t-tests comparing participants' evaluations of communication in a language that is not their first language and their evaluations of communication in general with the four target groups (patients, colleagues, doctors, and other professionals), showed that foreign language communication was experienced by all target groups as significantly more problematic than communication in general $(p<.001$; see Table 1). An independent-samples t-test comparing retail pharmacists to everyone else, for both communicative situations for each target group (total of 8 tests), did not indicate a significant effect of current employment on communication problems.

Open-ended responses indicated that problems do arise from the practitioner's limited active vocabulary knowledge (Appendix A.2), pronunciation (producing and understanding - see Appendix A.6), difficulties with grammar in the foreign language (Appendix A.7), the absence of labels for certain conditions in the patient's mother tongue, the patient's illiteracy (Appendix A.8), and the language used being different from the interactant's mother tongue (Appendix A.1). Language-discordant situations are reported (by some) to be especially problematic in discussions about "sensitive areas" (for example, "when one has to explain inserting a vaginal preparation or a suppository"), where it is "difficult to be professional and diplomatic". In line with the biographical finding that most practitioners seem to be fluent in English and/or Afrikaans but not in African languages ${ }^{9}$, problems seem to occur when patients do not speak or understand English or Afrikaans (see Appendix A.9).

\subsubsection{Elements of importance while communicating with patients/clients}

Collectively, respondents indicated that "good listening skills" was the most important element while communicating with patients or clients (see Table 2). Other important elements included: being able to ask questions, knowledge of the patient's language and culture (i.e. knowledge about culturally-sensitive topics), being able to speak clearly, being able to respond reflectively, summarising what has been said, using simple vocabulary, and using simple sentences.

\footnotetext{
${ }^{9}$ Upon initial review, this seems mainly due to infrequent use, not due to unwillingness to learn.
} 
Table 2: Pharmacists' ratings of the importance of aspects of professional communication in a second/foreign language (from most to least important)

\begin{tabular}{lcc}
\hline & $M$ & $S D$ \\
\hline Good listening skills & 1.50 & 0.75 \\
Being able to ask questions & 1.59 & 0.76 \\
Using simple vocabulary & 1.66 & 0.81 \\
Using simple sentences & 1.67 & 0.80 \\
Being able to speak clearly & 1.68 & 0.77 \\
Empathy & 1.70 & 0.88 \\
Being able to respond reflectively & 1.71 & 0.78 \\
Appropriate eye contact & 1.74 & 0.87 \\
Summarising what has been said & 1.76 & 0.84 \\
Knowledge of their culture (sensitive topics, etc.) & 1.82 & 0.84 \\
Appropriate body language & 1.83 & 0.88 \\
Knowledge of their language & 1.84 & 0.85 \\
Appropriate use of silence during communication & 1.89 & 0.88 \\
Direct face-to-face communication (no phone, no interpreter) & 1.95 & 1.02 \\
Experience talking with them & 2.12 & 0.88 \\
Knowledge of cultural hierarchies & 2.23 & 0.96 \\
Knowledge of jargon and abbreviations & 2.34 & 1.01 \\
Using pictures or drawings & 2.45 & 1.08 \\
\hline
\end{tabular}

The open-ended, qualitative comments suggest that there is a need especially for language and communicative support in history taking (covering people skills and passive vocabulary knowledge) and ensuring the correct use of medication (covering passive and active knowledge of instructions and compliance) - see Appendix A.10-11. For this part of the questionnaire, respondents also voluntarily described how to solve communication problems on the job (see Appendix A.12).

\subsubsection{Perceived barriers for conducting effective communication}

When asked which of the following aspects were barriers for conducting effective communication in their profession, participants selected lack of knowledge $(67.69 \%)$, lack of skills $(63.85 \%)$, lack of resources (41.54\%), lack of interest $(30.77 \%)$, and low priority $(26.92 \%)$. Open-ended responses pointed to time constraints and lack of respect or engagement. Some examples communicated verbally by the respondents were as follows: "Lack of respect from other medical proffessions for my proffesion"; "Bad salaries of certain staff and not investing in training staff which strains their development. Bad attitude of certain people who does not accept responsibility for their function in the process"; "pharmacists have lost the passion for pharmaceutical care and the provision of information and education". Also, a lack of self-awareness was raised as a barrier, as noted by one respondent: "Lack of realising what contributes to a communication problem, and how our individual styles impact on the patient." 


\subsection{Pedagogical needs}

The pedagogical component of the needs inventory required respondents to indicate on a 5point Likert scale to what extent they would like to learn the listed languages and topics if they "were to take a communication course for pharmaceutical purposes" (with lower scores indicating a greater desire to learn a given language or topic).

Respondents were also asked to indicate what they think the focus of a syllabus for effective oral communication for pharmacists should be. The options provided were: speech functions, cultural functions, social functions, language structures, communication principles and structures, active student participation, or other. Another question asked participants to indicate how a communication course for pharmacists should be taught: face-to-face, with printed course materials, with online materials, with mobile support, or other. For this question, endorsing multiple options was allowed. Finally, participants were asked who should teach these kinds of courses: a pharmacist specialising in communication for pharmacists, a language specialist working in the area of LSP (pharmacy), a pharmacist in collaboration with a language specialist, or someone else. Respondents could also add comments.

\subsubsection{Learning, training, teaching}

Collectively, respondents preferred to learn isiZulu for pharmaceutical purposes $(\mathrm{M}=2.40, \mathrm{SD}$ $=1.27)$, and were also open to isiXhosa $(\mathrm{M}=2.84, \mathrm{SD}=1.32)$, Afrikaans $(\mathrm{M}=3.09, \mathrm{SD}=$ 1.31), Setswana $(M=3.02, S D=1.30)$, Sesotho $(M=3.07, S D=1.26)$, and more English $(M$ $=2.91, \mathrm{SD}=1.49)$.

In terms of content, respondents indicated that they wanted to learn more about nearly all the topics suggested: communicative strategies $(\mathrm{M}=1.92, \mathrm{SD}=0.98)$, how to deal with drug abuse and prevention $(\mathrm{M}=1.90, \mathrm{SD}=0.94)$, how to use simple and clear sentences $(\mathrm{M}=1.89, \mathrm{SD}=$ $0.99)$, how to recognise patients' emotions $(\mathrm{M}=1.96, \mathrm{SD}=1.02)$, medical terminology in another language $(\mathrm{M}=2.03, \mathrm{SD}=1.09)$, cultural limitations regarding medication consumption (for instance, not taking medicine containing alcohol due to religious reasons; $\mathrm{M}=2.01, \mathrm{SD}=$ $0.90)$, alternative medicine $(\mathrm{M}=2.07, \mathrm{SD}=0.90)$, cultural customs and sensitivities $(\mathrm{M}=2.12$, $\mathrm{SD}=1.04)$, and cultural hierarchies $(\mathrm{M}=2.45, \mathrm{SD}=1.11)$.

Some open responses were quite emotionally-loaded, reflecting potential ongoing frustrations whilst communicating professionally: "useless to learn about patient's emotions and reflection etc. if you can't even penetrate their inaccurate ideas about health and can't communicate with them due to language barriers. I find it totally useless"; "My answers about cultural sensitivities and customs are based on my believe that cultures that oppress women or others (hierarchies) should not be respected- instead they should be the ones getting with the times. We are living in the $21^{\text {st }}$ century and I find these social norms and behaviours unacceptable and will not contribute to them in way, shape or form."

A syllabus for oral communication focusing on communication principles and structures was considered most important (64.86\% from 74 participants, same sample for the percentages to follow here), followed by a syllabus focusing on active student participation $(54.05 \%)$, speech functions (47.30\%), language structures (43.24\%), cultural functions (33.78\%), and social functions $(32.43 \%)$. One respondent requested that we "please start with first year students already with 
workshops". A majority of $64.86 \%$ indicated that a communication course for pharmacy students should be taught by a pharmacist in collaboration with a language specialist, followed by $20.27 \%$ recommending a language specialist working in the area of LSP (pharmacy), and $14.86 \%$ favouring a pharmacist specialising in communication for pharmacists. One respondent suggested adding "[...] a person knowing the culture of the target language group", thereby confirming the need for attention to the culturally-determined aspects of communication.

With regard to teaching methods, the majority (87.84\%) believed the course should be taught faceto-face. Having online materials $(36.49 \%$ ) was also considered important, but this could also be alongside printed course materials $(21.62 \%)$ and with mobile support $(21.62 \%)$. Time was mentioned as the "biggest constraint", and workshops were suggested "to reinforce behaviour".

\section{From theory to needs analysis to materials design}

\subsection{Summary of the needs analysis}

Whereas most respondents seem to manage professional language-discordant encounters, they experience communication in a foreign language to be significantly more problematic than communication in general. Importantly, problems in second, third, fourth, and even fifth languages are reported to be mainly due to infrequent use rather than unwillingness to learn. The qualitative data suggest that support is needed especially in African languages, and less so in English and Afrikaans. However, the quantitative results suggest that the respondents prefer a communication course for pharmaceutical purposes mainly for isiZulu and isiXhosa and, to a (nearly equally) lesser extent, for English, Afrikaans, Setswana and Sesotho.

Learning materials should cover basic vocabulary (as active vocabulary is limited), scientific terminology and simple explanations for patients/clients, sensitive topics (for instance, related to sexual issues, treatments applying to the genital area, etc.), pronunciation tips, grammatical support, and how to ask questions. Problems arising from different cultural and historical backgrounds, strict adherence to professional hierarchies, different intellectual abilities or illiteracy also highlight the importance of a more general communication module. This component should encourage creativity whilst communicating (for instance, using gestures to support language), active listening, checking of one's communication partner's and one's own understanding of the exchanged messages (by, e.g. summarising), a focus on patient-centred healthcare, awareness of the impact of one's own communication skills and style on others, and, ideally, an introduction to alternative medicine and cultural customs. Two main areas in practitioner-patient interaction to which these materials should apply are history taking (covering people skills and vocabulary) and ensuring the correct use of medication (covering passive and active knowledge of instructions and compliance).

The majority of participants indicated that they prefer a communication course for pharmacy students to be taught face-to-face by a pharmacist in collaboration with a language specialist. Alongside printed course materials, online materials and mobile support are also valued. Most importantly, learning materials should be to-the-point and easy to apply, as most practitioners have limited time to study and to interact with patients/clients, colleagues and other professional contacts. 


\subsection{Formulating goals and objectives}

The needs analysis indicates that it is important for practitioners to use language effectively in a professional context and, in particular, to: (i) develop general communication skills; (ii) learn about their clients' cultures, especially when related to (the use of) medicine; and (iii) learn medical terminology alongside other profession-specific vocabulary in different languages. Based on the participants' perceived needs, the goals and objectives outlined in the following sections were established to help students to communicate successfully with both clients and other healthcare professionals in English, Afrikaans, isiZulu, isiXhosa and Setswana.

\subsubsection{Overall learning goals}

Pharmacists should be able to communicate with their clients and colleagues in a professional and socially appropriate way at a proficiency level where they can: (i) understand the main ideas of complex text on both concrete and abstract topics, including technical discussions in their field of specialisation; (ii) interact with a degree of fluency and spontaneity that makes regular interaction with native speakers quite possible without strain for either party; and (iii) produce clear, detailed text on a wide range of subjects and explain a viewpoint on a topical issue, giving the advantages and disadvantages of various options in each case.

Upon completion of a language and communication programme designed for pharmacy students, learners should:

(i) Have developed general social communication skills (including assessing the other person's specific communicative needs and preferences, recognising their emotions and responding accordingly, producing simple and clear utterances, etc.) in order to improve their conversations with clients and other healthcare professionals they are likely to encounter as a practitioner;

(ii) Be aware of the existence of cultural differences regarding medical practice and medicinal consumption among their clients, and take these differences into account while communicating with clients and providing healthcare; and

(iii) Be able to understand, "translate" and explain medical terminology and key professionspecific phrases in English and Afrikaans, and effectively use the Communication for Professionals communication tools in isiZulu, isiXhosa and Setswana.

\subsubsection{Specific outcomes}

While learning or training with and using the proposed language and communication programme in the clinical setting, students should meet a number of specific outcomes in English, Afrikaans, isiZulu, isiXhosa, and Setswana with an accuracy of at least $80 \%$ in the test phase. They should be able to:

(i) Identify different sorts of communication barriers (linguistic, medical, social, emotional, religious, political, etc.) in day-to-day healthcare scenarios;

(ii) Overcome communication barriers by constructing a conversation which minimises potential discrepancies between their own intellectual, social and moral features and those of their clients by, for instance, adjusting their language use to the client's needs by correctly asking for information and/or through active listening; 
(iii) Identify the cultural and language backgrounds of their clients while providing healthcare in order to cater for every client's healthcare needs, beliefs, and preferences; and

(iv) Use basic communication and interactive strategies so they can greet their clients, ask about their personal situation (concerning their visit), and say goodbye to them.

Moreover, for Afrikaans and English, learners should be able to:

(i) Ask clients simple and clear questions about their medical history by making use of the consultation timeline;

(ii) "Translate", describe, and explain medical terminology that is frequently used in the pharmacy (for instance, related to the (consumption of the) medicine that is being sold);

(iii) Prescribe a pharmaceutical product without language mistakes;

(iv) Give clear instructions on how to use the pharmaceutical product (medical terminology together with reference to everyday life and potential dangers);

(v) Give a 50-word oral response to a hypothetical question asked by a client, with the former deemed as satisfactory by at least $80 \%$ of an exam jury made up of experienced professionals; and

(vi) Report with $80 \%$ accuracy the medical history and healthcare needs of a client to a colleague.

Since isiZulu, isiXhosa and Setswana can be identified or labelled as "less widely taught", the approach to learning how to function professionally in any of these languages should be more pragmatic both content-wise (focusing on a limited selection of highly significant and frequent language functions) as well as pedagogically, where strategic and "just-in-time" learning may be most effective. To this end, during contact sessions, learners will be introduced to the online/mobile materials and how to use them in a language-discordant clinical setting. By adopting a blended approach, the programme not only advocates online/mobile and lifelong learning, it also supports the development of an autonomous attitude which learners will need in order to become the competent professionals they strive to be.

In terms of the specific outcomes for the African languages, the learners should be able to:

(i) Identify socially and culturally appropriate phrases in the initial and final phases of the encounter;

(ii) Adequately use the verbal and non-verbal interaction patterns required in these phases;

(iii) Be familiar with the bilingual consultation timeline for history taking and medicinal consumption as presented in the Communication for Professionals online materials;

(iv) Effectively make use of this consultation timeline in the clinical setting by, specifically, identifying the phase of the encounter, isolating the question(s) to be asked, gauging the best language in which to ask it, asking the question, and ensuring appropriate feedback and successful communication.

\subsubsection{Outlining the programme}

Even though the different languages addressed in this programme serve different functions and will have to be learned in different degrees, the look and feel as well as the outline of the 
programme remain the same for all languages, even though the syllabus is most comprehensive for English and Afrikaans and covers 10 topics. The learning line is gradual, from easy to more complex, but also caters for repetition of more challenging language content. Figure 1 is a screenshot taken from the Communication for Professionals - Pharmacists online materials for English, which were informed by the theoretical insights as explained above, as well as by the needs, wants and wishes of practicing pharmacists.

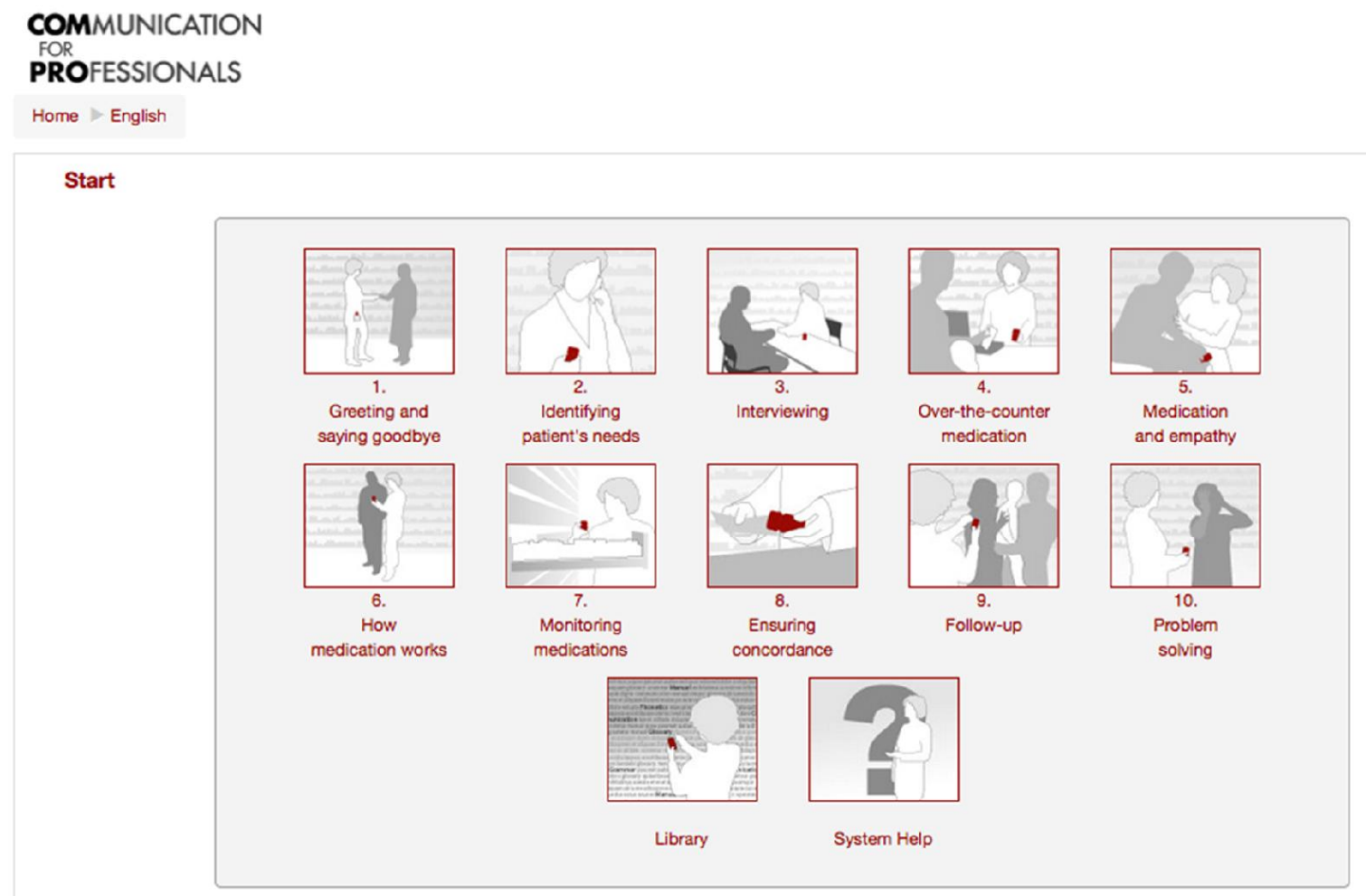

Figure 1. Screenshot of the Communication for Professionals - Pharmacists start-up page with various topics, Library and System Help

The topics cover different types of content captured in scenarios. Listed below are a few examples to illustrate the interactive communicative content as well the content embedded in the consultation timeline:

1. Greeting and saying goodbye: Opening and closing an encounter

- Greeting a patient

- Identifying a patient

- Social talk

- Starting counselling

- $\quad$ Saying goodbye

2. Identifying patient's needs: Selling prescription medication

- The prescription

- Products used

- Verifying the doctor's advice 
- Contacting the family doctor over the telephone

- Early demand for a repeat prescription

3. Interviewing: Patient assessment

- Identification

- History taking

- Medication overview

- Current medical conditions

- Medication record

\subsubsection{Preview of a scenario}

Each topic is developed around short scenarios. A scenario represents the communicative functions performed by a pharmacist in clinical practice. For example, the second topic "Identifying patient's needs" contains five scenarios which are elaborated on in training at the levels of sound, meaning, language form (grammar), and communication. Figure 2 is a screenshot taken from the Communication for Professionals - Pharmacists online materials, providing an illustration of a scenario in the second topic. The first scenario in the second topic deals with getting a prescription filled.

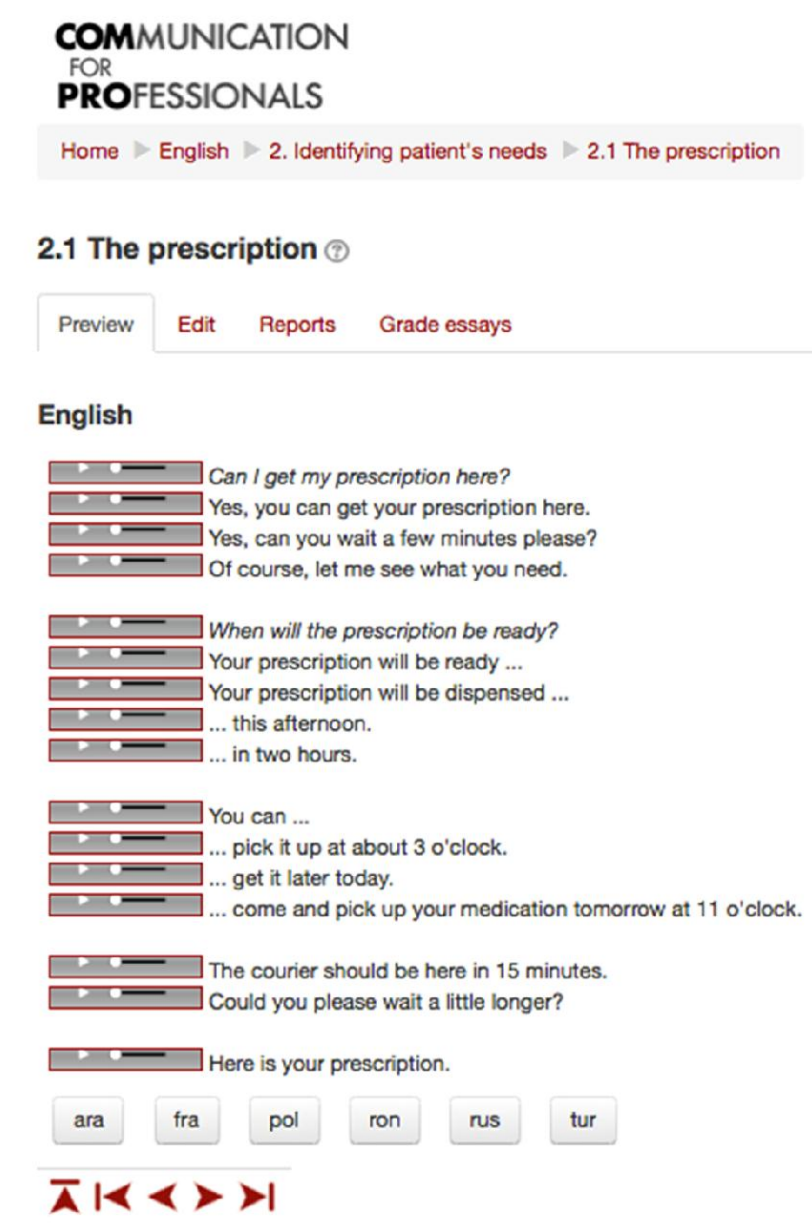

Figure 2. Screenshot of a scenario from Communication for Professionals - Pharmacists 


\subsubsection{Training materials}

Learners can practise the different components of the target language as well as the communicative functions that are required in a clinical setting. Communication for Professionals - Pharmacists provides practice for sounds, word meaning, language structure as well as communication skills. Figure 3 is a screenshot taken from the online materials, providing an illustration of one of the communication exercises. Learners have to complete utterances and listen for hints and tips. After completing an exercise, they can check their answers, and a mark and feedback for remedial training is then provided. The feedback will point learners to either the scenarios, the wordmaps (new words presented in semantic fields or word families) or support materials in the Library.
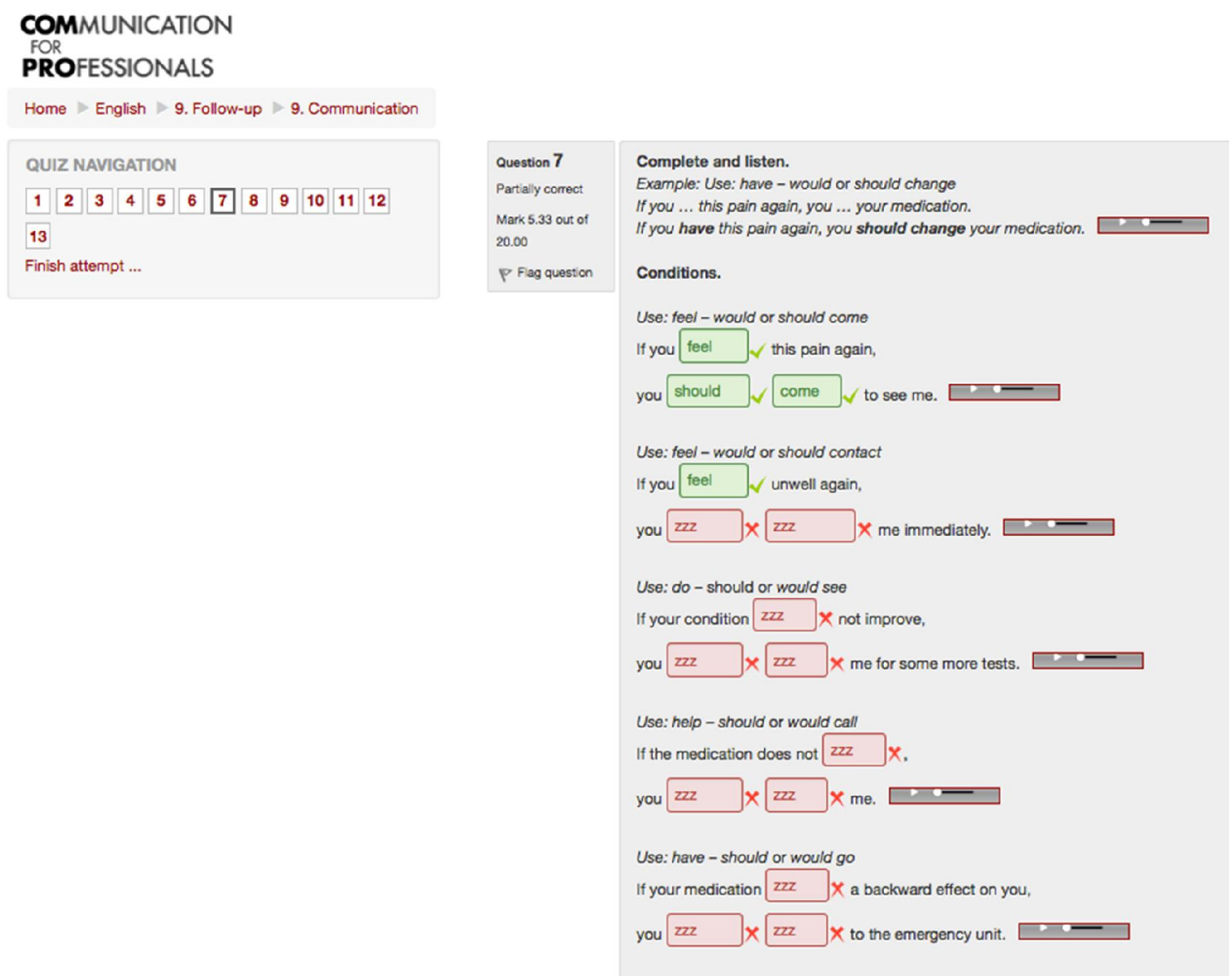

Figure 3. Screenshot of a completed and corrected exercise (without feedback) from Communication for Professionals - Pharmacists

\subsubsection{Additional materials: The Library}

In order to support learning and realise the required communicative outcome, additional materials are provided in the Library. Since pharmacists generally have an analytical learning style, background information to language components can stimulate the learning process. To 
this end, the Library contains the following tools: a pronunciation manual with videos showing air stream mechanisms, articulation and medical terms exemplifying the sounds; a wordlist with audio examples and translations to access the scenarios easily; a user-friendly and contextualised grammar overview as a support to access the topics; and communication strategies and tips which should facilitate effective interaction in the foreign language and culture (Figure 4).

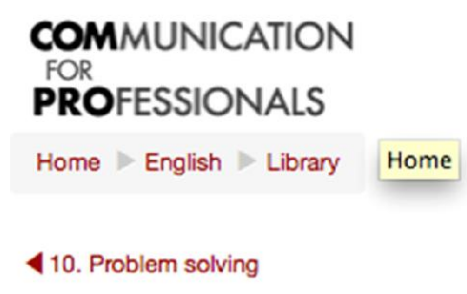

Library

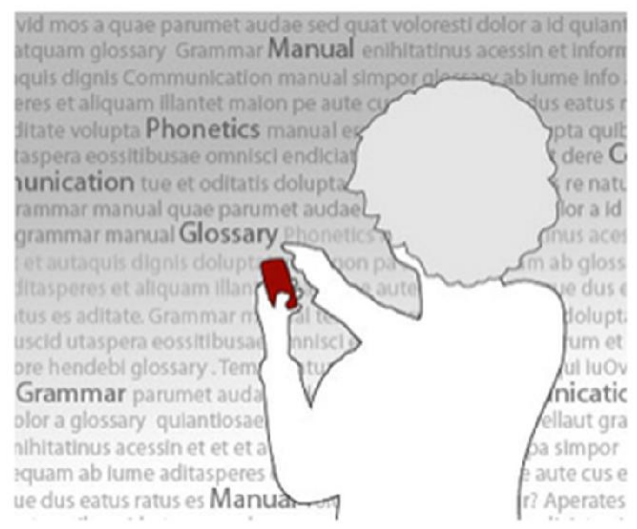

Hello. I'm Myriam Sano, pharmacist and practitioner. Welcome to the reference section.

Here you can find different manuals, the Glossary and System Help Documentation in different languages.

The Pronunciation Manual gives a complete inventory of

all sounds in English with videos on how to pronounce them.

All the examples are medical words.

The Grammar Manual is a complete grammar of the English language.

All the examples are medical and mostly taken from the scenarios.

You can click on the topics you want to study.

The Communication Manual contains strategies and tips on how to communicate with patients and colleagues.

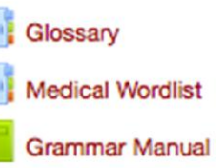

Figure 4. Screenshot of additional materials available in the Library of Communication for Professionals - Pharmacists

\section{Conclusion}

The guiding question for this article was: How can programme and materials design be informed by theory, and by drawing on pharmacists as experts-by-experience in intercultural professional communication? Through this study, we reported on how we managed the development process of a communication programme to be implemented at a South African 
university while drawing on the voices of stakeholders (i.e. teachers, students, alumni, professionals) engaged in pharmaceutical communication. In doing so, we identified in the first phase of the project a workable theoretical framework, i.e. Language for Specific Purposes. We then developed and conducted a needs analysis in the second phase of the project, the results of which were translated into a framework for syllabus and programme design. The ultimate goal of this process was to develop course materials to enhance (pre-)professionals' efficient functioning in a multilingual work environment where appropriate communication is of critical importance. We set out to investigate whether professionals could be relied upon to inform intercultural professional communication course content, and have never before arrived at such a rich database from which to work. This multilayered process may have the power to inform guidelines about responsible and effective functioning in a multilingual work environment where clear and transparent communication, more often than not, is a matter of life or death.

When performing a needs analysis, a vital component is an evaluation of the process of implementation. Since we have just concluded the conceptual phase of the analysis, our next step is to plan a usability study of the implementation of the course materials in which we will determine how learners evaluate the programme as a whole (both in contact teaching and online) and what the possible impact of this programme is on healthcare communication. A further research project following from this study will be the translatability and adaptability of the programme (materials and approach) into the African languages. Ultimately, we are interested in investigating equivalence among different programmes under the Communication for Professionals - Pharmacists umbrella.

\section{Acknowledgements}

We would like to thank Awie Kotze, Dean of the Faculty of Health Sciences at North-West University (NWU), for making this project happen. We are also grateful to Sandra van Dyk, Willem Basson and Mariëtta Basson (all from the NWU's School of Pharmacy) for their assistance and support with the refinement of the needs analysis. Wannie Carstens was of great value too in helping to put the project on track. Finally, our special thanks go to all the participating pharmacists for their time and energy invested in the project.

\section{References}

Ali, H.I.H and A.R.A. Salih. 2013. Perceived views of language teachers on the use of needs analysis in ESP materials writing. English Language Teaching 6(3): 11-19.

Basturkmen, H. and C. Elder. 2006. The practice of LSP. In A. Davies and C. Elder (Eds.) The handbook of applied linguistics. Oxford: Blackwell Publishing. pp. 672-694.

Beardsley, R.S., C.L. Kimberlin and W.N. Tindall. 2012. Communication skills in pharmacy practice. Philadelphia: Lippincott Williams and Wilkins.

Berbyuk Lindström, N. 2008. Intercultural communication in health care. Non-Swedish physicians in Sweden. Gothenburg Monographs in Linguistics, Vol. 36. Gothenburg: University of Gothenburg. 
Berwick, R. 1989. Needs assessment in language programming: From theory to practice. In R.K. Johnson (Ed.) The second language curriculum. New York: Cambridge University Press. pp. 48-62.

Bonk, C.J. and C.R. Graham (Eds.) 2006. Handbook of blended learning: Global perspectives, local designs. San Francisco: Pfeiffer Publishing.

Brown, J.D. 1995. The elements of language curriculum. Boston: Heinle and Heinle Publishers.

Carkin, S. 2005. English for academic purposes. In E. Hinkel (Ed.) Handbook of research in second language teaching and learning. New York: Lawrence Erlbaum. pp. 85-98.

Communication for Professionals. 2014. Communication solutions where and when you need them. Available online: http://www.comforpro.com/ (Accessed 1 August 2014).

Dudley-Evans, T. 2001. Developments in English for specific purposes: A multi-disciplinary approach. Cambridge: Cambridge University Press.

Fatihi, A.R. 2003. The role of needs analysis in ESL program design. South Asian Language Review XIII(1/2): 39-59.

Flowerdew, L. 2013. Needs analysis and curriculum development in ESP. In B. Paltridge and S. Starfield (Eds.) The handbook of English for specific purposes. Malden: Wiley-Blackwell. pp. 325-346.

Gasiorek, J. and K. Van de Poel. 2012. Divergent perspectives on language-discordant mobile medical professionals' communication with colleagues: An exploratory study. Journal of Applied Communication Research 40(4): 368-383.

Gatehouse, K. 2001. Key issues in English for specific purposes (ESP) curriculum development. The Internet TESL Journal 7(10). Available online: http://iteslj.org/Articles/ Gatehouse-ESP.html (Accessed 15 September 2014).

Hutchinson, T. and A. Waters (Eds.) 1987. English for specific purposes: A learning-centred approach. Cambridge: Cambridge University Press.

Lepetit, D. and W. Cichocki. 2002. Teaching languages to future health professionals: A needs assessment study. In M.H. Long (Ed.) Second language needs analysis. Cambridge: Cambridge University Press. pp. 105-124.

Long, M.H. (Ed.) 2005. Second language needs analysis. Cambridge: Cambridge University Press.

McDonough, R.P. and M.S. Bennett. 2006. Improving communication skills of pharmacy students through effective precepting. American Journal of Pharmaceutical Education 70(3): 58.

Richards, J.C. 2001. Curriculum development in language teaching. Cambridge: Cambridge University Press. 
South African Government. 1997. Constitution of the Republic of South Africa (Act 108 of 1996). Available online: http://www.gov.za/documents/constitution/1996/a108-96.pdf (Accessed 31 July 2014).

South African Government. 2013. Census 2011: Statistical release. Available online: www.statssa.gov.za/publications/P03014/P030142011.pdf (Accessed 29 July 2014).

Van de Poel, K. 2009. Medics on the move: The effect and effectiveness of on-the-workfloor language training. Available online: http://www.ou.nl/Docs/Campagnes/ICDE2009/Papers/ Final_paper_135vanderPoel.pdf (Accessed 7 July 2014).

Van de Poel, K. 2011. From multidimensional needs to language training for mobile professionals: An interdisciplinary approach. In S. De Wannemacker, G. Clarebout and P. De Causmaecker (Eds.) Communications in information and computer science. Berlin/Heidelberg: Springer Verlag. pp. 70-84.

Van de Poel, K. and T. Brunfaut. 2010. Medical communication in L1 and L2 contexts: Comparative modification analysis. Intercultural Pragmatics 7(1): 103-130.

Van de Poel, K. and C. Fourie. 2013. A critical approach to the development of blended medical communication training materials. Stellenbosch Papers in Linguistics Plus 42: 333-351.

Van de Poel, K. and J. Gasiorek. 2012a. Effects of an efficacy-focused approach to academic writing on students' perceptions of themselves as writers. Journal of English for Academic Purposes 11(4): 294-303.

Van de Poel, K. and J. Gasiorek. 2012b. Linguïstische noden van mobiele medische professionals. (Linguistic needs of mobile medical professionals.) In N. de Jong, K. Juffermans, M. Keijzer and L. Rasier (Eds.) Papers of the ANELA 2012 Applied Linguistics Conference. Delft: Eburon. pp. 194-201.

Van de Poel, K., E. Vanagt, U. Schrimpf and J. Gasiorek. 2013. Communication skills for foreign and mobile medical professionals. Berlin/Heidelberg: Springer Verlag.

Warrington, S.D. 2005. The 'what', 'why' and 'how' of needs assessment for adult EFL learners. Journal of Asia TEFL 2(4): 56-66. 


\section{Appendix A: Qualitative data on communication (The data have been reproduced here with their original spelling.)} (1) Problems due to lack of fluency in $2^{\text {nd }} / 3^{\text {rd }} / \ldots$ languages,
but rarely in English or Afrikaans

(2) Problems with specific terminology in $2^{\text {nd }} / 3^{\text {rd }} / \ldots$ language

(3) Problems due to different cultural/historical backgrounds

(4) Problems due to different semiotics
"Cannot ask questions to ascertain symptoms or give instructions to people who cannot understand English or Afrikaans"

"I do not understand any African languages as sometimes needed in private sector (when the patient only understand African languages especially in rural areas). Fortunately it is easy to get someone who can interpret the patients concerns so that the patient can be helped accordingly."

"Although in my current job I do not use other South African languages, I worked in retail pharmacy for 5 years and there were some instances were knowledge of an African Language would have been beneficial. Some people are able to better explain problems in their own language, people coming to suburban pharmacies from squatter camps may not speak English"

"English is easier to communicate in - in the manufacturing environment all process and equipment are trained in English"

"I am fully conversant in both English and Afrikaans. These two languages are the dominant languages in business"

"I work in a rural setting and English is not spoken or understood by most patients"

"I can instruct Patient and Klients in layman terms exceptionally well I have forgiotten the scientific terms that I studied because I do not converse in those terms anymore.."

"Medical terms are difficult to translate"

"Communication with professionals could be a slight problem if there were technical subjects to be discussed - I would battle with eg medical terms due to lack of use in my present employment!"

"I have spoken so little Afrikaans since Community service, that it is difficult to come up with the right vocabulary, specifically when speaking with doctors and colleagues where requirements are more technical"

"I rarely deal with the public and almost everyone else speaks English. On the odd occasion, someone prefers to speak Afrikaans which I understand easily, but do not speak fluently. The scientific nature of the work makes it even harder to find the words to express myself. However, I make it clear that if the other person understands English, that we each speak our own language, and that works."

Comment on general communication problems:

"Patient has different cultural values and norms, making it hard for me to medicate"

\section{Comments on language-discordant communication} problems:

"explaining allergies, side effects and drug interactions with traditional medicines"

"I advise on legal/ethical/professional matters. With the vast differences in cultural and language backgrouds amongst many, it is not always possible to communicate the finer nuances of professional legal/ethical problems."

"Most patients who speak an african native language like tswana and sotho etc are very problematic to communicate with, not because they can't speak english, but because english that they use mean something different to them than we understand. Also, these people's cultural health beliefs are a great obstacle. They believe if you're tired, you must have "gal". "Gal" can mean constipation, or it can just mean that (even if you have regular bowel movements) your bowels must be "cleaned". They therefore come to you 
(5) Problems due to other's "refusal" to speak English

(6) Pronunciation problems

(7) Grammatical problems

(8) Problems due to patient's illiteracy

(9) Mainly problems in African languages

(10) Need for support in history taking

(11) Need for support in ensuring the correct use of medication

(12) Problem solving on the job complaining about "gal", which will have them get something for constipation, but they are not constipated, they are tired, which means they probably can have blood sugar problems, vitamin B deficiency, etc. "Gal" is also the description given for phlegm, sinus, diahrroea, vomiting etc. They believe these symptoms are due to "gal", which means the bowels need to be emptied. Huge obstacle."

"Many African languages don't have the typical concepts that is prevalent in european languages, e.g. the word allergy does not exist in Xhosa. So I have to ask this the long way round: 'have you taken any medicines before that caused you to itch or that you know of that is bad for you?'”

"when the doctor or patient refuses to be spoken to in English. I would then have to skeap in afrikaans

"When a doctor refuses to speak English and only wants to communicate in Afrikaans. This is rare though. I have only encountered this once in my professional life."

"Pronunciation of certain conditions and drugs is the most common problem"

"tenses"; "Six cases, 2 genders and neutral and different endings depending on singular or plural"

"Portugese customers from Mozambique - Illiterate people from rural community who needs instructions how to take their medicine"

"COUNSELLING PATIENTS WHO ARE ILLITERATE"

"Knowing the persons education back ground will help you to know how to approuch the person/client"

"Communicating with patients and colleagues in a black language about medical conditions and describing symptoms."

"Understanding and communication to a patient that does not understand english / afrikaans"

"problem in understanding what people want and sickness explanation"

"Patients get comfortable when communicated in the language they understands and most of them open up more."

"words sound similar to my ear and I can give the patient wrong medication. [...] I cannot get a full history to make my diagnosis"

"Proving credibility and being sure that the patient is understanding important information"

"My main problem is to try and make the patient listen so that he/she knows when and how to take their medicine eg.telling a patient to take a tablet 3 times daily,then when making sure that they know the dosage asking them how many times a day they should take their pills they answer twice a day.I have been in pharmacy for $40 \mathrm{yrs}$ and never been able to resolve this problem."

"Confirm if all instructions were understood and ask for the patient to repeat them"

"Reflecting back to make sure that I have understood and interpreted all the information it correctly."

"A negative reaction from a client/patient would be a blank stare this is when the counsellor or assistant is called to translate"

"Face to face communication is ideal but an interpreter is important to me when I have a foreign client. Also, telephonic communication is also important for convenience" "Google translator on iPhone is very useful."

"All the necessary communication is left to the pharmacist assistants"

"If it's an older patient, be prepared to listen to the story of her life etc" 


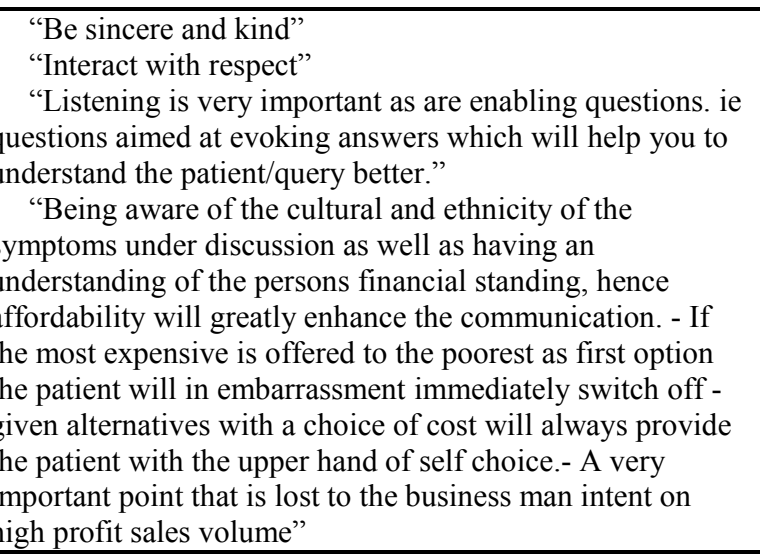

